\title{
Racionalizando las políticas sociales en América Latina: el papel de la gestión
}

\section{Ernesto Cohen* y Rolando Franco**}

\section{Debilidades de la política social en América Latina}

Un problema crucial de la política social en América Latina hoy es la escasa proporción de recursos que se destinan a los más pobres, independientemente del financiamiento de que ella disponga. Algunos ejemplos bastan para justificar esta afirmación. Sólo un dólar de cada $\$ 7.83$ gastado en programas sociales llegaba, en 1987, al 20\% peor ubicado en la distribución del ingreso, en Chile, país que durante varios años había realizado importantes esfuerzos por focalizar el gasto en ese sector poblacional (HaindL, Buvinic e Irarrazaval, 1989). Asimismo, un informe confidencial de un organismo internacional afirmaba, respecto a otra nación de la región, que suprimir todos los programas sociales en ejecución no provocaría cambio alguno en las condiciones de vida del $50 \%$ más pobre.

Dos razones pueden explicar estos hechos. Una es que la política social ha estado dirigida fundamentalmente a otros grupos sociales; otra, la ineficacia de la institucionalidad vigente, de la forma en que se diseñan y se implementan los programas y las filtraciones que se producen en ellos.

En el aspecto institucional conviene recordar que el Estado latinoamericano brinda servicios sociales a través de una red fragmentada, donde es común la duplicación de funciones y servicios, lo que conduce al desperdicio de recursos y a que sólo una porción marginal de los mismos llegue a los beneficiarios. Cálculos realizados para algunos programas han mostrado que la transferencia efectiva no supera el $5 \%$ de su presupuesto total, mientras que el resto se destina a gastos burocráticos o se filtra hacia otros grupos.
La relación inversión-impacto está mediada por múltiples decisiones. El resultado depende tanto de que se haya realizado una selección adecuada de los beneficiarios (focalización), como de la accesibilidad económica, social y cultural que los presuntos beneficiarios tengan al programa y, asimismo, de que la cantidad y calidad de los bienes y servicios ofertados sean correctas.

Un error ampliamente difundido que contribuye a que la relación postulada anteriormente no se dé en la práctica tiene lugar en programas de alimentación escolar en los cuales se proporcionan raciones alimentarias similares a todos los niños de un mismo grupo etario. En realidad, el programa debería proponer intervenciones orientadas a solucionar el problema de la desnutrición que, obviamente, no afecta por igual a todos los alumnos. Si los destinatarios del programa no tienen daño nutricional inicial, el impacto de las raciones consumidas, en el mejor de los casos será nulo; en el peor, conducirá a que los niños terminen presentando problemas de obesidad. Dado que, en general, los recursos están mal asignados puede no haber efectos positivos o, si los hay, ellos seguramente serán menores a los que pudieron alcanzarse de haber concentrado esfuerzos y recursos en los verdaderamente desnutridos.

Se incurre asimismo en errores de diseño derivados de no considerar la estrategia de sobrevivencia de las familias. Así, hay casos comprobados en que niños provenientes de familias con necesidades básicas insatisfechas empeoran su situación nutricional durante el período en el que están recibiendo las raciones del programa nutricional. La razón estriba en que tales programas entregan un complemento calórico-proteico que no pretende reemplazar la alimentación recibida en el hogar. Pero no se informa de esas características a las familias beneficiarias, por lo cual las más pobres - ante la carencia de alimentos que enfren$\tan$ - excluyen a quienes comieron fuera del hogar. 
Los programas sociales sufren también dificultades derivadas de perseguir objetivos secundarios que afectan la posibilidad de alcanzar los principales. En este sentido, merece recordarse que los recursos de algunos Fondos de Inversión Social se destinan a cubrir lo necesario para "inversión", sin tomar en cuenta el financiamiento de los costos recurrentes (de operación), o estableciendo que ellos son de la responsabilidad de los beneficiarios. Con ello se busca promover el compromiso con la obra. Sin embargo, cuanto más pobre es la comunidad menor será la probabilidad de que pueda afrontar tales gastos. Otro problema surge de la decisión según la cual los Fondos actúan a partir de la iniciativa de los interesados, con el riesgo de atender sólo a los que conocen esa disponibilidad de recursos públicos y tienen capacidad de moverse en los vericuetos administrativos, excluyendo así a los más pobres. Lo mismo sucede cuando se establece que la preparación de los proyectos es responsabilidad de los eventuales beneficiarios que carecen de capacidad para ello. Asimismo, puede apreciarse que la inercia burocrática tiende a colocar los recursos de inversión en regiones de fácil acceso, donde la población no es la más carenciada.

La implementación también juega un papel central para el logro del impacto. En dicha fase a menudo se producen situaciones que generan efectos perversos, incluso en programas bien diseñados. Un ejemplo: distribución de los alimentos de algún programa, pretendiendo paliar alguna crisis, entre más personas que las previstas originalmente. La consecuencia es que todos reciben raciones más pequeñas, que no cumplen los requerimientos calórico-proteicos mínimos. Cuando además se producen filtraciones que favorecen a quienes no son parte de la población meta, se reduce todavía más el impacto del programa.

Por todo lo anterior, puede afirmarse que en América Latina (y esto puede hacerse extensivo con seguridad al resto de los países en dęsarrollo y, ¿a lo mejor?, a muchos programas en ejecución en naciones desarrolladas) se desconoce el desempeño de los programas y proyectos en los que se plasman las orientaciones de política social y, sobre todo, se ignora el impacto que tales acciones producen sobre la población. En general, no se sabe si tales programas sirven o no sirven; si sirven, cuánto sirven y en qué grado se justifican; quiénes se aprovechan realmente de sus beneficios y a quiénes perjudica su realización.

En consecuencia, un objetivo central de la política social contemporánea es su racionalización, aumentando la eficiencia en la utilización de recursos y la eficacia de los programas y proyectos.

\section{El papel de la gestión}

Las políticas sociales se traducen operacionalmente en programas y proyectos ${ }^{1}$. Por ello, la formulación y evaluación de éstos constituyen el aspecto básico -usualmente omitido, sin embargo- en el proceso de asignación de recursos, al mismo tiempo que la gestión debe ser la dimensión central a tener en cuenta durante su operación. Evaluación y gestión, por tanto, son las vías para la racionalización del gasto público social.

Esta racionalización es fundamental no sólo en situaciones en que disminuyen los recursos y aumentan las necesidades, como las vividas en América Latina durante la crisis de los años ochenta y las que actualmente enfrentan México y otros países de la región, sino que también es importante en coyunturas de auge económico, en las que surgen nuevas necesidades sociales que requieren mayores recursos financieros.

Por lo mismo, aumentar la eficiencia en la utilización del financiamiento disponible y la eficacia de las acciones implementadas para enfrentar problemas de naturaleza social constituye una exigencia permanente, que plantea requerimientos específicos en la gestión de los programas y proyectos sociales.

En un ámbito donde suele postularse la conveniencia de trasladar métodos de gestión privada novedosos al sector público, conviene reflexionar sobre las diferencias fundamentales que existen entre el ámbito empresarial y el de los programas sociales, para saber hasta qué punto es adecuada esa transferencia y de qué manera hay que enfrentar las especificidades de los que aquí interesan.

El problema fundamental de una empresa privada tiene que ver con su eficiencia, lo que lleva a que el control de los costos de operación sea el objetivo central de su administración.

En los programas y proyectos sociales, por el contrario, el problema central es la eficacia en el logro de sus fines. En algunos casos pueden ser eficientes, pero no consideran al impacto como la función objetivo perseguida.

Se aducen varias razones para explicar esto. La primera y más difundida sostiene que los proyectos sociales tratan con intangibles y que, por lo tanto, sus resultados son de carácter cualitativo y no medibles. Esto, en el mejor de los casos, es una verdad a medias. Como se ha dicho: "El desarrollo de la personalidad integral como objetivo de la escuela, ciertamente es cosa inalcanzable. Por lo menos, no es tarea de este mundo. Pero, enseñar a leer a un niño cuando ha terminado tercer grado, de ningún modo es imposible y es una actividad que puede medirse fácilmente y con bastante precisión" (DruCKER, 1990, p.97).

De hecho, los objetivos generales de las políticas se expresan habitualmente en términos cualitativos. Así, por ejemplo, es normal que se hable de "lograr el desarrollo económico y social", "mejorar el sector agrícola", o "promover la integración social". Mientras tales objetivos generales no sean traducidos en objetivos específicos no será posible precisar metas, tiempos, ni actividades orientadas a alcanzarlos y, por tanto, ni se podrá diseñar la gestión que más les acomoda. 
De ahí, que una condición tanto para la formulación como para la gestión de un programa o proyecto es definir con claridad los objetivos específicos que pretende alcanzar.

Los proyectos con objetivos vagos no pueden ser evaluados. Esto es, no pueden utilizarse metodologías que buscan la optimización del logro de fines, cuando éstos no son lo suficientemente precisos. De forma análoga, una eficiente gestión de programas y proyectos presenta la misma exigencia. Si no existe un fin establecido con toda precisión no hay forma de asignar racionalmente los recursos. Como decía Séneca, "Para quien no sabe adónde va, cualquier viento es favorable".

Existe otra cuestión de relevancia mayor. La función de una empresa privada es maximizar el beneficio que se realiza en el mercado. Si la empresa no es monopólica debe entregar un bien o un servicio por el que los consumidores estén dispuestos a pagar. La tasa y volumen de ganancias que la empresa obtiene es la medida de su éxito.

Los programas y proyectos sociales, tal como funcionan generalmente hoy, son implementados por el sector público que opera de manera monopólica o cuasimonopólica, entrega los bienes y/o servicios en la cantidad que estima conveniente y los financia con recursos del erario público asignados por razones administrativas, políticas o derivadas de presiones corporativas. Los usuarios, en la generalidad de los casos, carecen de alternativas reales para escoger y sus necesidades sentidas no son las que guían la asignación de los recursos y el diseño de los programas. Por ello, a diferencia de lo que sucede con las empresas privadas el grado de logro de los objetivos y metas perseguidos por la organización no tiene por juez al mercado.

Esto exige establecer otra forma de medir los resultados obtenidos por los programas y proyectos. Sólo la evaluación de impacto ex-post podría determinar en qué medida el proyecto justifica el presupuesto que se le ha asignado. Lo que sucede, en cambio, es que si los problemas para enfrentarlos cuando se ha formulado y aprobado el proyecto no se solucionan o, incluso, se agravan, tiende a aumentarse (o, por lo menos, a tratar de elevar) el presupuesto asignado. De ahí que resulte posible que mientras más ineficaz sea un proyecto, mayor sea la probabilidad de que sobreviva e, incluso que aumente los recursos de que dispone.

\section{La especificidad de los proyectos sociales}

Los proyectos, productivos o sociales, se elaboran para satisfacer necesidades. Si quienes las sienten son económicamente solventes, demandarán los bienes o servicios y será el mercado quien resuelva. Quienes carecen de recursos constituyen una "demanda social", a la que deben proveer los satisfactores de sus necesidades en forma gratuita o a un precio que no es el de mercado.

Los programas y proyectos sociales que se inscriben en el marco de la superación de la pobreza buscan la satisfacción de dicha demanda social.

\section{Eficiencia y eficacia}

Una estrategia orientada a superar las limitaciones existentes en política social debe incorporar tanto el análisis de la eficiencia como el de la eficacia de los proyectos ${ }^{2}$.

Eficiencia es la relación entre los costos de los insumos y los productos (servicios o bienes) obtenidos. Cuando está definida la cantidad de productos a entregar, se busca minimizar los costos por unidad de producto. En cambio, cuando es fijo el gasto total en que puede incurrirse, se persigue maximizar el producto, teniendo ambas definiciones un significado equivalente.

El análisis de la eficiencia se lleva a cabo tanto ex-ante como durante la operación del proyecto. No debe confundirse con el monitoreo o seguimiento, que se limita a comparar los resultados con las metas establecidas en la programación.

La eficacia de un proyecto es el grado en que éste logra sus objetivos en un período de tiempo determinado, sin considerar los costos. Cuando se eliminan los efectos contextuales y se consideran los efectos netos del proyecto, se la llama impacto.

Este análisis se realiza de manera independiente del orientado a evaluar la eficiencia operacional (evaluación de procesos).

La razón por la cual estos análisis se realizan separadamente tiene que ver con la cantidad de relevamientos de información que requieren. La reprogramación sólo requiere uno para determinar la "línea de base", que es un corte transversal a partir del cual se elabora el diagnóstico, que permite proponer soluciones. En cambio, los modelos aplicables a la evaluación de impacto exigen una "línea basal" de diagnóstico y una "línea final" de resultados. La comparación entre ambas permite verificar la magnitud de los cambios atribuibles al proyecto.

Es importante acotar que la línea de base, normalmente asociada al diagnóstico, puede establecerse en cualquier momento del proceso de operación. Por otro lado, la línea final no necesariamente exige que el proyecto haya concluido. Es, en realidad, otro corte transversal que proporciona información equivalente a la facilitada por la línea de base. El tiempo que debe mediar entre la obtención de la información inicial y la requerida por esta segunda línea depende de la naturaleza de las transformaciones perseguidas (nutricionales, de salud, educacionales, etc.) y del grado de sensibilidad de los indicadores que se utilizan en su medición. Estos indicadores deben ser seleccionados por expertos en las áreas sustantivas del proyecto. 
La evaluación de impactos se lleva a cabo durante la operación y a la finalización del proyecto e, inclusive, después que éste ha terminado, una vez transcurrido el tiempo requerido para que se manifiesten todos sus efectos. Sobre la base de las evaluaciones de impacto ex-post pueden realizarse estimaciones ex-ante de proyectos análogos.

\section{Análisis de Impacto}

Hay factores no relacionados con la eficiencia operacional que pueden dificultar y hasta impedir que muchos proyectos sociales alcancen los fines que persiguen. A dar cuenta de ello se orienta el análisis o evaluación de impacto. Por impacto se entiende la medida en que un proyecto, en función de sus objetivos, transforma alguna parcela de la realidad. Ello exige considerar explícitamente los efectos netos del proyecto eliminando los cambios derivados del contexto.

La evaluación de impacto determina en qué medida el proyecto ha alcanzado sus objetivos, qué cambios ha producido en la población beneficiaria y cuáles fueron sus efectos secundarios (previstos y no previstos).

Se puede concluir, en suma, que el análisis de impacto es una condición necesaria pero no suficiente para la evaluación y gestión de los proyectos sociales. Siendo su justificación medir el grado de eficacia de un proyecto, no considera explícitamente los costos en que se incurre para alcanzarla. Esto supone asumir que los recursos disponibles son ilimitados. Se trata de un efecto de la clásica división disciplinaria en las actividades de evaluación: el análisis económico está prioritariamente preocupado por la eficiencia, mientras que la eficacia ha sido tradicionalmente el interés de quienes trabajan en las áreas sociales sustantivas (nutrición, salud, educación).

\section{Jerarquía de objetivos}

En los proyectos, los objetivos se encuentran organizados en una estructura jerárquica en la que es necesario diferenciar, por lo menos, tres niveles.

a) Objetivos de actividades e insumos requeridos (¿qué necesita el proyecto?). Los insumos son los recursos de todo tipo (físicos, humanos, financieros, etc.) que se aplican a las acciones (actividades) destinadas a obtener los productos del proyecto.

b) Objetivos de productos (¿qué debe entregar el proyecto?). Son los resultados que se pretende obtener con los recursos asignados. Un proyecto de complementación alimentaria, por ejemplo, requiere insumos (tales como infraestructura, vajilla, alimentos, combustible y personal) para realizar la actividad central, que es la preparación de los alimentos que se entregarán al grupo objetivo. Las raciones alimentarias son los productos del proyecto.

El conocimiento disponible sobre la relación existente entre insumos y productos permite la programación y el monitoreo o seguimiento. La cantidad y combinación de los insumos necesarios para producir determinada cantidad de unidades de producto es el prerrequisito para asignar recursos al proyecto. Seleccionar la alternativa óptima es la finalidad del análisis de la eficiencia que permite escoger una opción técnicamente viable, que minimice el costo por unidad de producto. Sus resultados permiten programar la entrega de bienes o servicios. Al mismo tiempo, posibilita el seguimiento del proceso de implementación, determinando el grado de alcance de las metas, en los tiempos y a los costos previstos.

c) Objetivos de impacto (ipara qué se hace el proyecto?). El proyecto no se lleva a cabo para proveer raciones alimentarias. Estas son sólo un medio, que se entrega para disminuir la tasa de desnutrición existente en la población objetivo. Este es el objetivo de impacto.

En un proyecto privado, el objetivo de impacto es la magnitud del beneficio que percibe la empresa que lo lleva a cabo. Su equivalente en un proyecto social es la medida en que se logra el impacto previsto en la población meta del mismo.

La jerarquía de objetivos supone una estructura causal en la que las relaciones que vinculan a los insumos con los productos son normalmente conocidas. Su adecuado manejo permite que los proyectos sean eficientes, esto es, que generen los productos a costos mínimos merced a una asignación racional de los recursos disponibles.

Pero habitualmente la conexión causal entre productos e impactos es una hipótesis no explicitada y casi nunca validada. Esto puede llevar a que, inclusive, los proyectos eficientes puedan ser totalmente ineficaces y carecer de impacto.

En los proyectos privados, el mercado castiga severamente la ineficacia. Por lo tanto, la gestión de esos proyectos tiene siempre a la ganancia (eficacia) como orientación básica. En los proyectos sociales, la administración se restringe, en el mejor de los casos, al análisis y control de la eficiencia. Pese a su importancia, se olvida del impacto.

¿Por qué la administración de proyectos excluye habitualmente el análisis del impacto? Pueden sugerirse varias razones:

i) Habitualmente se asume una relación causal de carácter lineal entre objetivos de producto y de impacto. Así, por ejemplo, se cree que la entrega de raciones alimentarias a la población objetivo producirá naturalmente una disminución en las tasas de desnutrición.

ii) El impacto, como magnitud del cambio producido en la población objetivo, es función de la implementación del proyecto y de otras variables sobre las cuales el proyecto no ejerce influencia alguna. En el caso ya citado, la medida del cambio nutricional es resultado de las actividades del proyecto, pero también de la influencia de otros factores (que pueden denominarse "externos") tales como la variación en la capacidad adqui- 
sitiva de la población objetivo, el cambio en los precios relativos de los alimentos, el acceso potencial a otros programas que cumplen funciones complementarias, etc.

iii) Si bien existen metodologías que permiten aislar el efecto de las variables exógenas y así determinar la magnitud del efecto neto del proyecto, suele argumentarse que, dado el costo de su aplicación, estos recursos debieran destinarse a incrementar la cobertura del proyecto (aunque no se sepa si sus acciones están produciendo el resultado buscado).

iv) Los gerentes de los proyectos sociales son contratados para velar por la eficiencia en la utilización de los recursos que les son asignados. Centran su actividad, por lo tanto, en las relaciones previstas entre los insumos que se proveen y los productos programados y realmente obtenidos. Esto quita la responsabilidad por el impacto de las funciones gerenciales y traslada el problema de la eficacia a la alta dirección que carece de elementos de juicio para introducir modificaciones al proyecto.

\section{El compromiso gerencial}

El compromiso que asume el gerente con los objetivos de impacto del proyecto es una variable clave para el éxito de la gestión.

El siguiente es un ejemplo que ilustra la afirmación anterior. Se trata de la evaluación de un proyecto de desarrollo rural integral en Brasil, llamado Polo Nordeste, dirigido a una población objetivo de pequeños productores rurales. Estos cultivaban algodón, de tres variedades (fibra larga, fibra media y fibra corta), plantadas en proporciones semejantes en cada unidad de producción, en una microrregión con marcadas variaciones anuales en la precipitación pluvial.

La gerencia del proyecto llegó a la conclusión de que, dadas las condiciones climáticas y las características del suelo, convenía generalizar el cultivo de algodón de fibra larga, de mayor precio en el mercado, incorporando el desmotamiento del mismo, lo que permitiría incrementar sustancialmente el valor agregado y, por tanto, el ingreso de los agricultores.

Para tal efecto, dispuso realizar tareas de extensión orientadas a permitir la gradual transición entre la lógica de cultivo prevaleciente y la recomendada. Sin embargo, debió enfrentar una cerrada oposición de los agricultores, que no estaban de acuerdo en concentrarse en el algodón de fibra larga, pese a las promesas de mayor rentabilidad que generaba.

El impasse se mantuvo hasta que el gerente comprendió las razones del rechazo al cambio. La lógica campesina podía traducirse en una matriz como la que a continuación se presenta. En las filas, se tiene la precipitación pluvial -que, como se dijo, era variable- clasificada en alta, media y escasa. En las columnas aparecen los tipos de cultivo (algodón de fibra larga, media y corta). Cada tipo de cultivo exige diferente intensidad de lluvia: mucha para el algodón de fibra larga; poca para el de fibra corta.

Recordando que los agricultores dividían sus parcelas aproximadamente en tercios para cada tipo de cultivo, las filas de la matriz muestran la producción posible que cada campesino obtenía en función de la precipitación pluvial. En todos los casos había producción, independientemente de la cantidad de lluvia. La función objetivo perseguida por los campesinos era minimizar riesgos, dado que la no existencia de producción hubiese implicado para ellos la pérdida de la tierra que era su único bien.

El objetivo postulado por la gerencia del proyecto partía de una lógica diametralmente opuesta: pretendía maximizar el beneficio. Estimaba que el riesgo era pequeño y resultaba viable asumirlo.

Pero cuando el gerente del proyecto comprendió que resultaba inabordable plantar sólo algodón de fibra larga, aunque fuera el óptimo desde el punto de vista de la rentabilidad, alteró su estrategia e incorporó como restricción la subsistencia del sistema de policultivo existente. Se dedicó entonces a optimizar la situación de base, esto es, a incrementar el valor agregado, mediante la incorporación de desmotadoras para los distintos tipos de algodón.

\begin{tabular}{|c|c|c|c|}
\hline & Algodón de fibra larga & Algodón de fibra media & Algodón de fibra corta \\
\hline Precipitación alta & Producción alta & Producción media & Producción baja \\
\hline Precipitación media & Producción media & Producción alta & Producción media \\
\hline Precipitación baja & Producción baja & Producción media & Producción alta \\
\hline
\end{tabular}


De este ejemplo se desprende con claridad que el compromiso de la gerencia con el impacto del proyecto fue crucial para hacerlo viable, adaptándolo a la realidad campesina.

El dilema que se plantea en la gestión de los proyectos sociales es que el compromiso gerencial resulta un componente esencial para lograr los objetivos de impacto, pero no es exigible.

Por otra parte, tampoco es razonable buscar personas excepcionales, porque es difícil que se encuentren disponibles en el mercado laboral. El desafío fundamental entonces es cómo conseguir que los proyectos sociales tengan una estructura de gestión que permita que las funciones gerenciales puedan ser desempeñadas por individuos promedio con una formación adecuada.

\section{El papel de los usuarios}

Tal como se apuntó, los usuarios de los proyectos sociales constituyen un grupo con necesidades insatisfechas sin capacidad de adquirir los satisfactores en el mercado. No constituyen demanda efectiva sino "social". Pese a ello, su participación en todas las etapas del ciclo de vida del proyecto es relevante para la eficiencia y la eficacia del mismo.

Un estudio de la Agencia para el Desarrollo Internacional del Gobierno de los Estados Unidos de América (USAID), realizado en 1975, buscó identificar los elementos que explicaban el éxito o fracaso de proyectos de desarrollo rural integral financiados por el Banco Mundial. Analizó 36 llevados a cabo en Africa y 35 realizados en América Latina.

Algunas variables habitualmente consideradas importantes para el éxito de un proyecto de este tipo, como la alfabetización de la población objetivo, la distancia a un camino permanente, la calidad del medio físico para la agricultura, el número de parcelas por extensionista, la experiencia anterior de los campesinos con este tipo de proyectos, etc., resultaron ser irrelevantes en la gran mayoría de los casos para explicar el éxito.

En cambio, se comprobó que era decisiva la participación de los beneficiarios tanto en el diseño como durante la implementación del proyecto, en una situación tal que ni los administradores del proyecto ni los usuarios del mismo tuvieran toda la capacidad de decisión (COHEN y FranCO, 1992).

Por otro lado, sin tomar como marco de referencia efectos tan globales que puedan expresarse como "éxito" o "fracaso" del proyecto, existen otras experiencias que son claros indicadores de la incidencia que la participación tiene sobre la eficiencia y la eficacia de los mismos.

En la evaluación del Programa de Promoción Social Nutricional de Argentina, realizada en 1985, se pudo verificar que el costo de una ración alimentaria difería sustancialmente entre las provincias según hubiera o no participación comunitaria.
Se desprende de los ejemplos anteriores que resulta esencial que los programas sociales impulsen una estrategia participativa. Esto constituye un verdadero desafío para la gestión de los mismos. Vale la pena recordar que para avanzar en el camino de la participación, "la forma de hacer las cosas es tan importante como las cosas que se hacen" (GARCIa Huidobro, 1985).

En el campo de los proyectos sociales esto supone que su administración establezca relaciones horizontales con los usuarios para viabilizar procesos permanentes de reajuste entre las metas, las formas previstas de alcanzarlas y las necesidades de los beneficiarios, que se van modificando como resultado de un contexto cambiante.

La participación de los usuarios requiere el fortalecimiento de la capacidad de gestión a nivel local. Hay, por lo menos tres razones para ello (SCHEJTMAN, 1989).

a) Los objetivos de impacto perseguidos pretenden modificar una línea de base que describe el problema que justifica la existencia del proyecto. Se pretende, por ejemplo, satisfacer un déficit alimentario-nutricional que se expresa en tasas de prevalencia de la desnutrición. La distribución de la desnutrición en la región o provincia es poco relevante. Los únicos datos pertinentes son los del nivel local, que rescatan la especificidad del problema, los grados de libertad de las alternativas adoptadas por el programa y los recursos movilizables localmente.

b) La participación social es una estrategia que persigue que la comunidad sea un recurso. Para que este potencial se transforme en un verdadero insumo adicional, la comunidad no sólo debe percibir el problema sino también la capacidad transformadora del proyecto. Ello exige ámbitos territoriales que posibiliten la homogeneidad de los usuarios y de las soluciones a implementar.

c) La participación puede retroalimentar la gestión mediante el control social en la operación del proyecto. Esto es factible si la escala del proyecto resulta compatible con la comunidad receptora del mismo. Esto sólo se produce a nivel local.

\section{Hacia una mayor racionalidad de las políticas sociales}

El problema de la política social no es -o no es sólo- de carencia de recursos, pese a que sobre esto suele insistirse en exceso. Lo más importante es la baja eficiencia con que se los utiliza y el desconocimiento que se tiene respecto a la eficacia de las acciones que ellos financian. Es evidente que la única manera por la cual la política social puede coadyuvar al logro de la equidad y al desarrollo económico es elevando su racionali- 
dad, cambiando la forma en que se la ejecuta y logrando mayor certeza respecto a que sus resultados son positivos. Ello sólo puede lograrse evaluando lo que se viene haciendo y estableciendo mecanismos permanentes de seguimiento y evaluación de los programas y proyectos.

No tiene sentido insistir en que se entreguen más recursos para repetir lo que se viene haciendo que, como se ha visto, los resultados obtenidos, muchas veces no los justifican. Es necesario entonces modificar la institucionalidad y el financiamiento para facilitar la incorporación de la actividad de evaluación.

En el momento actual existen en América Latina multiplicidad de importantes programas, muchos de ellos financiados por la cooperación internacional, que han adoptado la lógica de los proyectos, obviamente diferente a la forma tradicional de hacer política social. Esas experiencias constituyen campos fértiles para comenzar a institucionalizar un proceso de racionalización creciente en las políticas sociales. Para ello sería conveniente tener presente los aspectos que se mencionan a continuación.

1. La gestión debiera orientarse fundamentalmente a alcanzar los objetivos de impacto que persiguen los programas y proyectos sociales.

2. Los programas y proyectos deben tener objetivos claramente definidos que permitan determinar metas y definir funciones y misiones de las organizaciones que los llevarán a cabo.

3. Deben fijarse prioridades que permitan la concentración de los recursos y el establecimiento de normas de rendimiento. Es fundamental establecer mínimos de resultados aceptables.

4. El proyecto debe retroalimentarse de la medición de la eficiencia y la eficacia que logra en su operación. La eficiencia se define como la relación entre los costos de los insumos y los bienes o servicios que constituyen los productos; la eficacia es la magnitud del impacto que los productos generan en la población objetivo a la que están destinados.

5. Dada la inexistencia de un mercado que permita la evaluación automática del éxito o fracaso del proyecto, se requiere efectuar evaluaciones costo-impacto, tanto ex-ante como ex-post.

En la etapa del diseño del proyecto es necesario especificar los indicadores de rendimiento que se van a utilizar para medir su desempeño, así como el sistema de información que los producirá. Durante la operación, corresponde calcular el coste específico por cada bien entregado o servicio prestado a los usuarios (costo por unidad de producto o servicio) para compararlo con la programación existente, si se está ante el caso de actividades sectoriales normatizadas o, alternativamente, evaluar su eficiencia relativa en función de la teoría y experiencias disponibles.

6. Si existen indicadores de rendimiento, las actividades y el personal que las realiza pueden ser evaluados en relación a los mismos. Las recompensas y castigos institucionalmente estructu- rados pueden frenar la arbitrariedad mediante reglas objetivas, que permitan reclamar legitimidad y consenso entre los miembros de la institución agente.

Las gratificaciones materiales (promociones, incrementos salariales) son estímulos que deben ser complementados por otros de carácter no material (participación en el diseño de los programas de trabajo, grados de libertad para la distribución de la carga horaria laboral) que marquen el desplazamiento del énfasis del tiempo de presencia del funcionario hacia lo que este produce durante el mismo (LANDELL-MiLLS, 1983).

7. Se debe estimular el involucramiento del personal con los fines de la organización. Al hacer más transparentes las medidas de éxito o fracaso, así como la contribución específica de los miembros de la administración a los resultados obtenidos, los incrementos de productividad adicionales van a depender de la moral de trabajo que la estructura institucional permita y estimule. Esto requiere cambiar el modelo tradicional burocrático predominante por otro más flexible, que aliente la innovación y donde lo heurístico tenga un claro predominio sobre lo normativo (KLIKSBERG, 1989).

8. Es deseable y previsible que los programas y proyectos sociales sean crecientemente integrados. Es evidente que las acciones de salud que se restringen a lo meramente curativo resultan poco eficientes y eficaces. Pero la prevención no puede contemplar sólo lo que típicamente se le asigna al sector (vacunación, educación para la salud, etc.), sino incorporar también los "condicionantes de salud" (saneamiento ambiental, agua potable, disposición de excretas, etc.) que tienen un peso determinante en los resultados que se pretenden alcanzar. Esto hace que los programas y proyectos sociales se tornen intersectoriales e interinstitucionales. Lo dicho plantea la necesidad de coordinar la gestión, tanto horizontal como verticalmente. La coordinación horizontal debe darse entre los distintos ministerios, el sector privado y la sociedad civil; la coordinación vertical debe producirse entre los distintos niveles espaciales en los que se organizan los programas (Estado nacional, región, provincia, municipio).

9. Se debe propiciar el compromiso gerencial con los objetivos de cambio perseguidos por el proyecto. Una tarea básica del gerente "es la creación de un conjunto auténtico que sea mayor que la suma de las partes, una entidad que rinda más que la suma de los recursos incorporados a la misma. Podenos formular una analogía: el director de una orquesta sinfónica, que con su esfuerzo, su visión y su liderazgo consigue que las distintas partes instrumentales se conviertan en la unidad viva de una ejecución musical. Pero el director tiene la partitura del compositor, no es más que el intérprete. El gerente es, al mismo tiempo, el compositor y el director" (DRUCKER, 1990, p.272).

Esta síntesis de la labor del gerente enfatiza que su función esencial es administrar eficientemente los recursos disponibles, orientando sus decisiones en función de los requerimientos del 
futuro inmediato y los del largo plazo. Los proyectos sociales tienen un requisito adicional: que el proyecto se oriente al impacto que se pretende lograr y su medición periódica sea la brújula orientadora de la gestión, tanto en su estructura interna (músicos e instrumentos) como en la conexión de la organización con su entorno.

10. Las condiciones anteriormente mencionadas son necesarias pero no suficientes para el éxito. Se debe contemplar además una participación activa de la población objetivo en la gestión del proyecto.

Esta cuestión suele dividir las opiniones en dos grupos contrapuestos. Por un lado, el de quienes propician la participación porque ésta se justifica por sí misma, o sirve de escalón para ir generando una organización creciente. Por otro, los que desconfian de ella, prefiriendo el juicio técnico como criterio de decisión por ser el más informado. A éstos hay que agregar los que argumentan que muchas veces la participación ha sido la excusa utilizada para sobreexplotar a los supuestos beneficiarios de proyectos sociales, por ejemplo, imputando un valor cero al costo de su trabajo en programas de autoconstrucción.

La participación es un recurso para lograr los fines del proyecto no por proporcionar mano de obra gratuita, sino porque permite inscribir los objetivos de los programas en la realidad local y viabiliza que los proyectos operen adaptándose a la misma. La experiencia muestra que la probabilidad de que los proyectos sean exitosos se acrecienta cuando existe una participación activa de los beneficiarios de los mismos.

Ello supone siempre una transferencia de poder. Pero no de todo el poder. Las decisiones estratégicas deben ser siempre negociadas con el grupo técnico del proyecto. Para ello, la estructura administrativa del mismo debe diseñarse de modo que los usuarios sean un agente normal en su operación.

Notas

*Coordinador del Programa Conjunto sobre Políticas Sociales, de la Organización de Estados Americanos (OEA) y la CEPAL.

*Director de la División de Desarrollo Social de la Comisión Económica para América Latina y el Caribe (CEPAL) de la Organización de las Naciones Unidas; y Coordinador de PROPOSAL.
${ }^{1}$ Los programas son conjuntos de proyectos que persiguen los mismos objetivos. Por ello, son formulados en grandes agregados de carácter nacional, regional o provincial. Los proyectos son las unidades mínimas de asignación de recursos, cuya localización espacial se da a nivel local.

${ }^{2}$ Para mayores desarrollos, puede verse COHEN y FRANCO (1992).

\section{Bibliografia}

Cohen, E. y R. Franco (1992): Evaluación de Proyectos Sociales, Siglo XXI, México.

DRUCKER, P.F. (1990): La gerencia. Tareas, responsabilidades y prácticas, El Ateneo Editorial, Buenos Aires,

GarCiA HutDoBRo, J.E. (1985): "Programas sociales solidarios y de educación popular. Una política emergenten, en Aspectos metodológicos de las politicas de desarrollo social, Santiago, ILPES/APSAL/ISUC.

GINESTAR, A. (1993): "Los cambios en la gestión pública", CICAP, OEA, Secreta- ría de la Función Pública, Buenos Aires.

Haind, BuvinIC e IrarRazÁval (1989): Gasto social efectivo, Universidad de Chile, Escuela de Economía/ODEPLAN, Santiago de Chile.

KLIKSBERG, B. (1989): "Cómo formar gerentes sociales? Elementos para el diseño de estrategias", en Cómo enfrentar la pobreza, Kliksberg, B. (compilador), Grupo Editor Latinoamericano, Buenos Aires.

LANDELL-MILLS, J. (1983): "La administración: un factor que fija límites al desarrollo", en Finanzas y Desarrollo, México. 